\title{
Increased EGFR Phosphorylation Correlates with Higher Programmed Death Ligand-1 Expression: Analysis of TKI-Resistant Lung Cancer Cell Lines
}

\author{
Kenichi Suda, ${ }^{1,2}$ Leslie Rozeboom, ${ }^{1}$ Koh Furugaki, ${ }^{3}$ Hui Yu, ${ }^{1}$ \\ Mary Ann C. Melnick, ${ }^{4}$ Kim Ellison, ${ }^{1}$ Christopher J. Rivard, ${ }^{1}$ Katerina Politi, ${ }^{4}$ \\ Tetsuya Mitsudomi, ${ }^{2}$ and Fred R. Hirsch ${ }^{1}$ \\ ${ }^{1}$ Division of Medical Oncology, University of Colorado Anschutz Medical Campus, 12801 E. 17th Ave. RC-1 South, Rm 8402J, \\ Aurora, CO 80045, USA \\ ${ }^{2}$ Division of Thoracic Surgery, Department of Surgery, Kindai University Faculty of Medicine, 377-2 Ohno-higashi, \\ Osaka-Sayama 589-0014, Japan \\ ${ }^{3}$ Product Research Department, Kamakura Research Laboratories, Chugai Pharmaceutical, 200 Kajiwara, Kamakura 247-8530, Japan \\ ${ }^{4}$ Department of Pathology, Yale University School of Medicine, 310 Cedar St. LH 108, New Haven, CT 06520, USA
}

Correspondence should be addressed to Kenichi Suda; ascaris@surg2.med.kyushu-u.ac.jp and Fred R. Hirsch; fred.hirsch@ucdenver.edu

Received 5 July 2017; Accepted 27 August 2017; Published 8 October 2017

Academic Editor: Ilary Ruscito

Copyright (C) 2017 Kenichi Suda et al. This is an open access article distributed under the Creative Commons Attribution License, which permits unrestricted use, distribution, and reproduction in any medium, provided the original work is properly cited.

\begin{abstract}
Despite the recent development of immunotherapies that target programmed death-1 (PD-1) or programmed death ligand-1 (PDL1) in non-small cell lung cancer (NSCLC) treatment, these therapies are less effective in NSCLC patients with epidermal growth factor receptor (EGFR) mutations. However, the molecular mechanisms underlying this lower efficacy of immunotherapies in EGFR mutant lung cancers are still unclear. In this study, we analyzed PD-L1 protein expression in lung cancer cell lines with EGFR mutations prior to and after acquisition of resistance to EGFR tyrosine kinase inhibitors (TKIs). We found that parental lung cancer cell lines harboring EGFR mutations showed negative (PC9 and H3255 cells) and positive (HCC827 cells) staining for PD-L1 by immunohistochemistry. Comparing PD-L1 expression between EGFR-TKI resistant cell lines and their parental cells, we found that increased phosphorylation of EGFR was related to increased expression of PD-L1. Increased phosphorylation of EGFR was accompanied by the T790M secondary mutation. Acquired resistance cells with MET amplification or EGFR loss both showed decreased phosphorylation of EGFR and decreased PD-L1 expression. Our results indicate that lung cancer cell lines with EGFR mutations (parental cells) do not harbor high PD-L1 protein expression. In addition, EGFR phosphorylation affects PD-L1 expression after acquisition of resistance to EGFR-TKIs.
\end{abstract}

\section{Introduction}

Activating mutations in the epidermal growth factor receptor (EGFR) gene define one of the most common molecular subtypes of non-small cell lung cancers [1]. EGFR tyrosine kinase inhibitor (TKI) monotherapies (gefitinib, erlotinib, or afatinib) are the first choice for these patients [1]; however, acquisition of resistance to these TKIs is almost inevitable after an average of 1 year [2]. A variety of resistance mechanisms have been identified including T790 M mutation, $M E T$ or ERBB2 gene amplification, small cell lung cancer transformation, and epithelial to mesenchymal transition (EMT) [2].

Osimertinib, a 3rd generation EGFR-TKI, is the appropriate second-line drug after acquisition of resistance to gefitinib, erlotinib, or afatinib if a rebiopsied resistant tumor proves the presence of an EGFR T790M secondary mutation [3]. However, cytotoxic chemotherapies are still the standard of care as second-line drugs for patients who do not have the EGFR T790M mutation [4]. Recent development of immunotherapies that target programmed death ligand-1 (PD-L1) or programmed death-1 (PD-1) has shown dramatic 
success in some lung cancer patients [5]. However, these immune-checkpoints inhibitors have shown poorer response rates and outcomes in patients with EGFR mutations compared to those with EGFR wild-type tumors $[6,7]$.

$\mathrm{PD}-\mathrm{L} 1$ protein expression has been pursued as a predictive marker for current immunotherapies. To elucidate the underlying mechanisms of this reduced effectiveness for immunotherapies in lung cancer patients with EGFR mutations, we performed the current study to analyze PD-L1 protein expression status, using the FDA approved detection kit system, before and after the acquisition of resistance to EGFR-TKIs in established cell lines harboring EGFR mutations.

\section{Materials and Methods}

2.1. Cell Lines, Reagents, and Generation of In Vitro Resistant Cell Lines. Human lung cancer cell lines used in this study were from the established collections in our labs or as reported in our previous studies [8-10]. PC-9 erlotinib resistant cells were established from PC-9 cells by stepwise exposure to erlotinib from $0.005 \mu \mathrm{M}$ to $5 \mu \mathrm{M}$ for about 4 months, and the clone named PC-9 ER clone 5 was isolated with PicoPipet (Nepa Gene, Chiba, Japan). Cells were cultured in RPMI1640 medium supplemented with 10\% fetal bovine serum (FBS) and 1x penicillin/streptomycin solution (Mediatech, Inc., Manassas, VA) at $37^{\circ} \mathrm{C}$ in a humidified tissue culture incubator with $5 \% \mathrm{CO}_{2}$. All experiments using acquired resistance cells were performed following the removal of drug exposure to avoid the direct effects of drugs on PD-L1 expression. IFN-gamma (Cell Signaling Technology, Dancers, MA) stimulation for 24 hours was performed to mimic an immune cell interaction.

2.2. Immunohistochemistry (IHC) Analysis. Formalin-fixed paraffin-embedded (FFPE) blocks were prepared from cell pellets to perform IHC analysis as previously described [11]. Briefly, cultured cells were gently harvested using Accutase ${ }^{\circledR}$ (Innovative Cell Technologies, Inc., San Diego, CA) and fixed with alcoholic formalin solution for 24 hours. Fixed cells were mixed with melted agarose solution, allowed to solidify, placed in the cassette, and submerged in $70 \%$ ethanol. Paraffin-embedding of the agarose cell pellet was performed at our pathology core lab. Antibody against PDL1 was purchased from Dako (22C3 pharmDx, Dako-Agilent Technologies, Carpinteria, CA). Staining was performed on a Dako Link 48 Auto-Stainer. PD-L1 staining was assessed using the $H$-score assessment.

2.3. Western Blot Analysis. All antibodies were purchased from Cell Signaling Technology (Danvers, MA). Total cell lysates were prepared, and immunoblotting was conducted as described previously [11]. Briefly, cells were cultured until subconfluent, rinsed with phosphate-buffered saline (PBS), lysed in sodium dodecyl sulfate (SDS) sample buffer, and homogenized. The total cell lysate $(10 \mu \mathrm{g})$ was subjected to SDS polyacrylamide gel electrophoresis (PAGE) and transferred to Immobilon-P polyvinylidene difluoride (PVDF) membranes (Millipore, Bedford, MA). After blocking with
$5 \%$ nonfat dry milk, membranes were incubated with primary antibodies, washed with PBS, and reacted with secondary antibodies (Cell Signaling Technology), and signals visualized using ECL reagent (Clarity, Bio-Rad, Hercules, CA) and film or detected by an ImageQuant Imager (GE Healthcare Bio-Sciences, Tokyo, Japan).

2.4. Flow Cytometry. PC-9 and PC-9 ER clone 5 cells were stained with the mouse monoclonal BV421-conjugated antibody to human PD-L1 (BD Biosciences, San Jose, CA) for flow cytometry analysis with the BD LSRFortessa cell analyzer and the BD FACSDiva software (BD Biosciences).

\section{Results}

3.1. PD-L1 Expression in Lung Cancer Cell Lines with EGFR Mutation. Initially, we screened for PD-L1 expression in parental lung cancer cell lines by IHC using the Dako 22C3 antibody. The efficacy of the 22C3 antibody was recently demonstrated in clinical trials $[12,13]$, and the analytical performance seems similar to two other clinically used PDL1 antibodies (Dako 28-8 and Ventana SP- 263 [14]). As shown in Figure 1, PC-9 cells (del E746_A750) and H3255 cells (L858R) were negative for PD-L1 IHC, and HCC827 cells (del E746_A750) showed positive expression for PD-L1 membrane staining ( $H$-score: 145$)$ that is weaker than that observed in other non-small cell lung cancer cell lines such as SW900, a lung squamous cancer cell line ( $H$-score: 210$)$. These results indicate that lung cancer cell lines harboring EGFR mutation do not have high $\mathrm{PD}-\mathrm{L} 1$ protein expression prior to EGFRTKI exposure.

3.2. EGFR Phosphorylation Levels after Acquisition of Resistance to TKIs Is Related to PD-L1 Expression Status. Initial evaluation for PD-L1 protein expression changes focused on comparisons between the parental HCC827 cells and a group of daughter cell lines with acquired resistance to EGFR-TKIs. Each daughter cell line exhibits different type of resistance mechanisms including MET gene amplification (HCC827ER), T790M mutation (HCC827EPR), MET gene amplification together with T790M mutation (HCC827 CNXR S1), and MET gene amplification with EGFR loss (HCC827 CNXR S4). As shown in Figures 2(a)-2(e), HCC827 daughter cells that have acquired resistance to EGFR-TKIs demonstrated various PD-L1 expression patterns including slightly decreased PD-L1 expression in HCC827ER and HCC827CNXR S4 cells ( $H$-scores: 125 and 120, resp.) compared with parental cells ( $H$-score: 145$)$. Meanwhile, both of T790M positive lines (HCC827EPR and HCC827 CNXR S1 cells) had higher PD-L1 expression ( $H$-scores: 220 and 190, resp.) compared with parental cells. These results were confirmed using western blot analysis (Figure 2(f)).

Because previous studies have observed a relationship between PD-L1 expression and EGFR activation in lung cancers (but no data regarding EGFR-TKI resistance and PDL1 expression) [15-18], we compared EGFR phosphorylation status between these cells. We observed that the acquired resistance cells with $\mathrm{PD}-\mathrm{L1}$ downregulation have decreased phosphorylation of EGFR (Y992 in HCC827ER cells and 


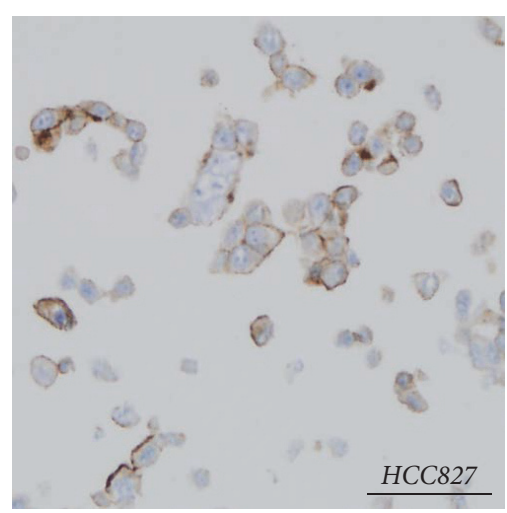

(a)

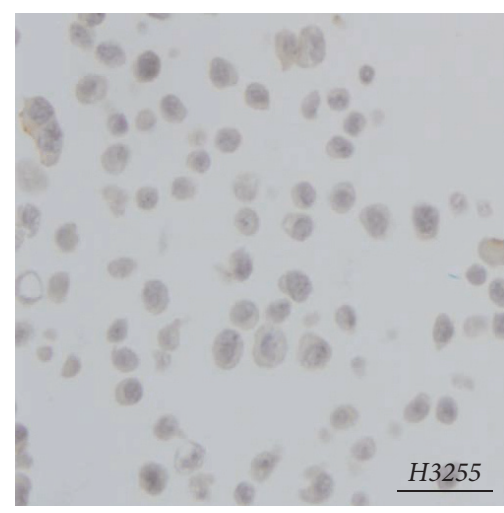

(b)

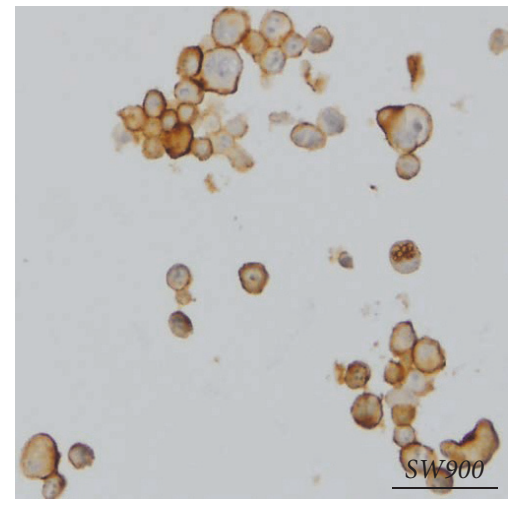

(d)

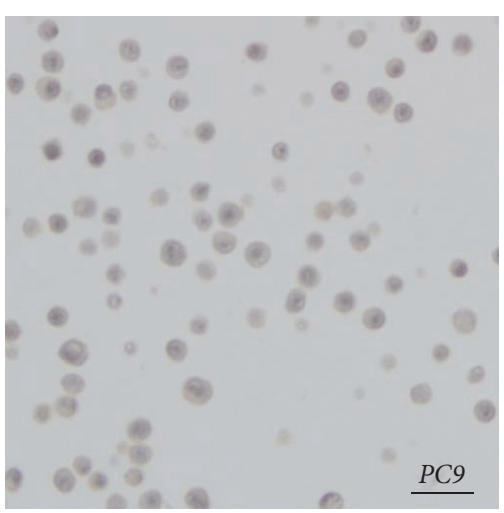

(c)

FIGURE 1: PD-L1 expression in parental lung cancer cell lines with EGFR mutations by IHC (Dako 22C3 antibody). (a) HCC827 showed positive staining ( $H$-score: 145 ). (b) and (c) H3255 and PC-9 cells demonstrate negative staining. (d) SW900 lung squamous cells with positive staining. Images at 20x, captured with a Olympus DP71.

all EGFR phosphorylation sites in HCC827CNXRS4 cells). On the other hand, resistant cells with upregulated PDL1 have increased phosphorylation of EGFR (Y992 and Y845 in HCC827EPR cells and Y1173 in HCC827CNXRS1 cells).

To generalize our findings in the HCC 827 series, we employed two other cell lines (PC-9 and H3255 cells) that developed acquired resistance to EGFR-TKIs via T790 mutation (PC-9ER clone 5, BRC1, 853\#10, and H3255XLR80 cells). As shown in Figure 3, three out of four cell lines had higher PD-L1 expression together with increased phosphorylation of EGFR compared to their parental cells. These data also indicated that no single phosphorylation site of EGFR is most responsible for increased PD-L1 expression. Only BRC1 cells showed similar EGFR phosphorylation and PD-L1 expression status compared with parental PC-9 cells.

3.3. Effect of IFN-Gamma Treatment on Parental and Resistant Cells. It has been established that cytokine signaling from the surrounding tumor microenvironment regulates PD-L1 expression in tumor cells [19]. To mimic an immune cell interaction, we treated HCC827 and their resistant daughter cell lines with IFN-gamma and measured PD-L1 expression. We observed that IFN-gamma treatment induced significant amount of PD-L1 protein in all cells examined (Figure 2(g)).

\section{Discussion}

In this study, we observed that cell lines with acquired EGFR-TKI resistance that harbor increased phosphorylation of EGFR, at any tyrosine residue, showed upregulation of PD-L1 protein expression. This finding is consistent with previous reports, which have observed that EGF stimulation upregulates PD-L1 expression or EGFR inhibition downregulates PD-L1 expression [15-18]. Each phosphorylation site of EGFR provides a binding surface for different substrate proteins, for example, GRB2 adaptor protein - Y1068 [20] or the SH2 domain of PLCgamma - Y992 [21]. However, all major downstream signaling pathways of EGFR, such as AKT serine/threonine kinase (AKT) - mechanistic target of rapamycin (mTOR) pathway [22], Janus kinase (JAK) - signal transducer and activator of transcription (STAT) pathway [23], or mitogen-activated protein kinase 1 (MAPK1) pathway [17], are reported to induce PD-L1 expression. Therefore, it would be reasonable that increased phosphorylation of any tyrosine residue of the EGFR is correlated with increased expression of PD-L1.

Our results may also provide for a possible explanation for lower efficacy of current immunotherapies in lung cancer patients with an EGFR mutation. We observed that PD-L1 protein expression is not high in parental cells with EGFR 


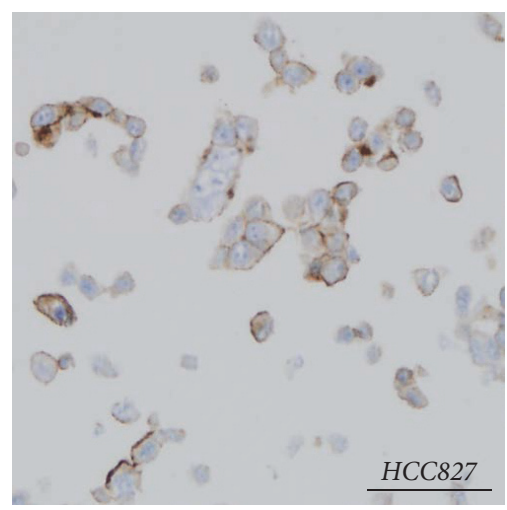

(a)

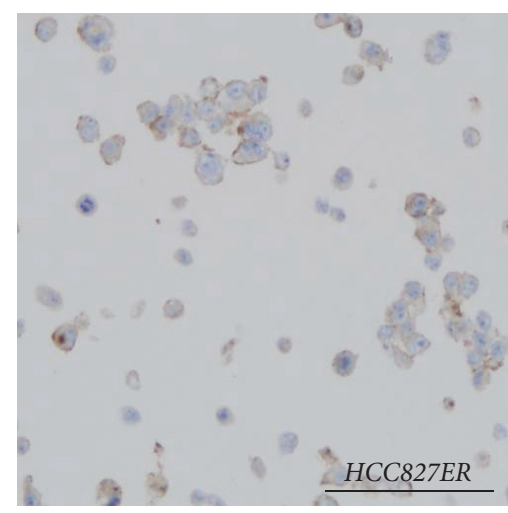

(b)

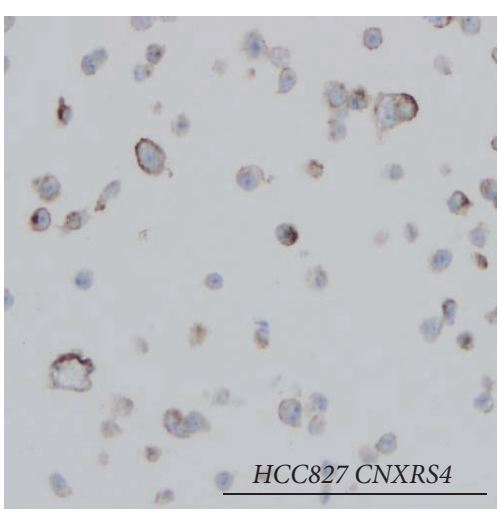

(c)

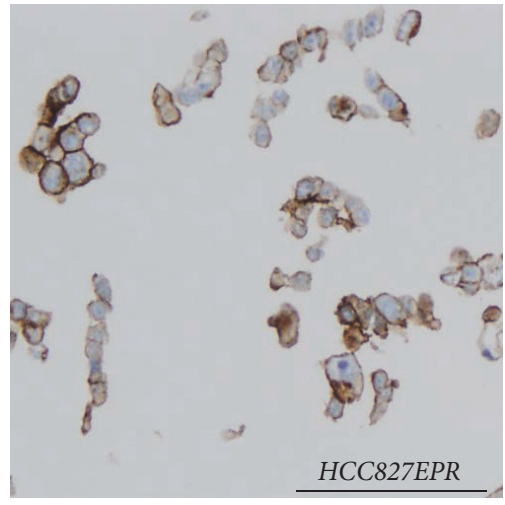

(d)

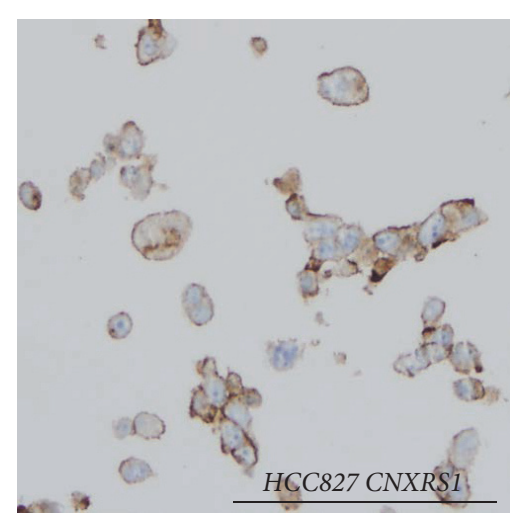

(e)

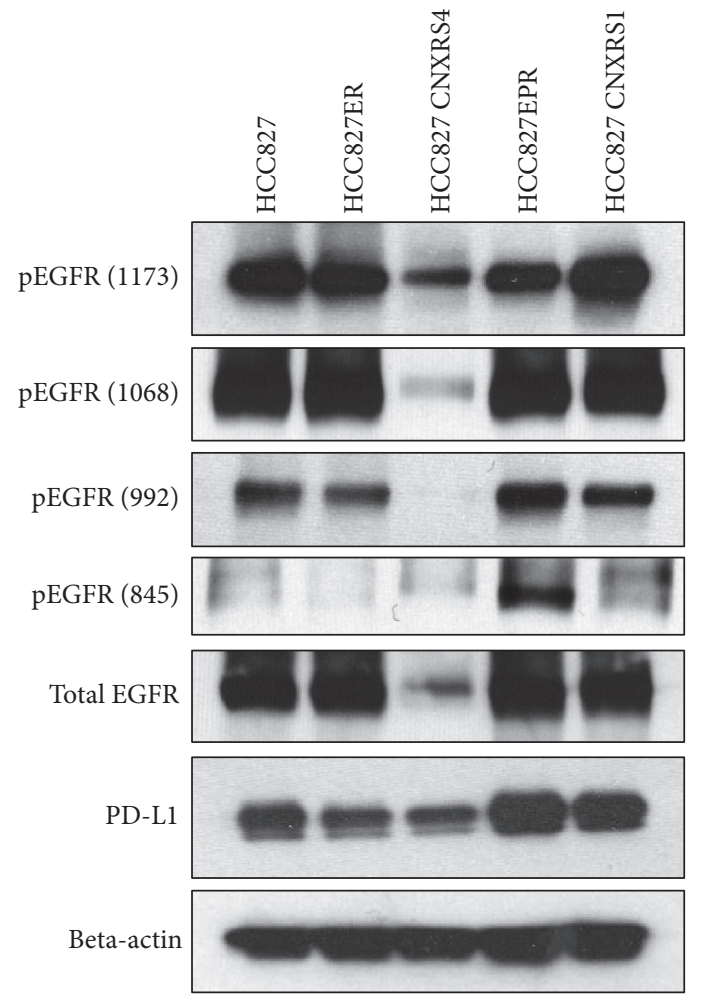

(f)

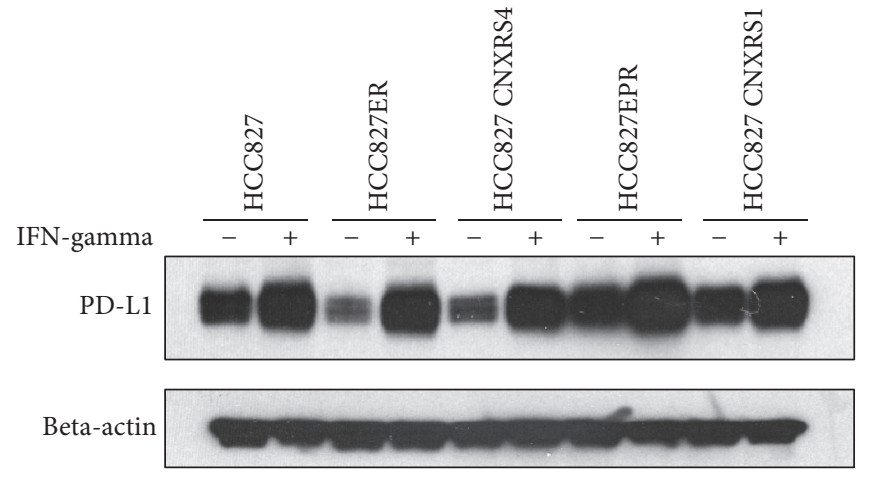

(g)

FIGURE 2: PD-L1 expression in parental HCC827 cells and their EGFR-TKI resistant daughter cells. (a)-(e) IHC staining for PD-L1 (Dako 22C3 antibody) in each cell line, showing decreased PD-L1 expression in HCC827ER and HCC827 CNXRS4 cells and increased PD-L1 expression in HCC827EPR and CNXR S1 cells. (f) Western blot analysis for PD-L1 and phosphorylation of EGFR. Beta-actin was used as loading control. (g) The effect of IFN-gamma exposure (100 U/ml, 24 hrs) for PD-L1 expression in HCC827 and acquired resistance daughter cell lines. 


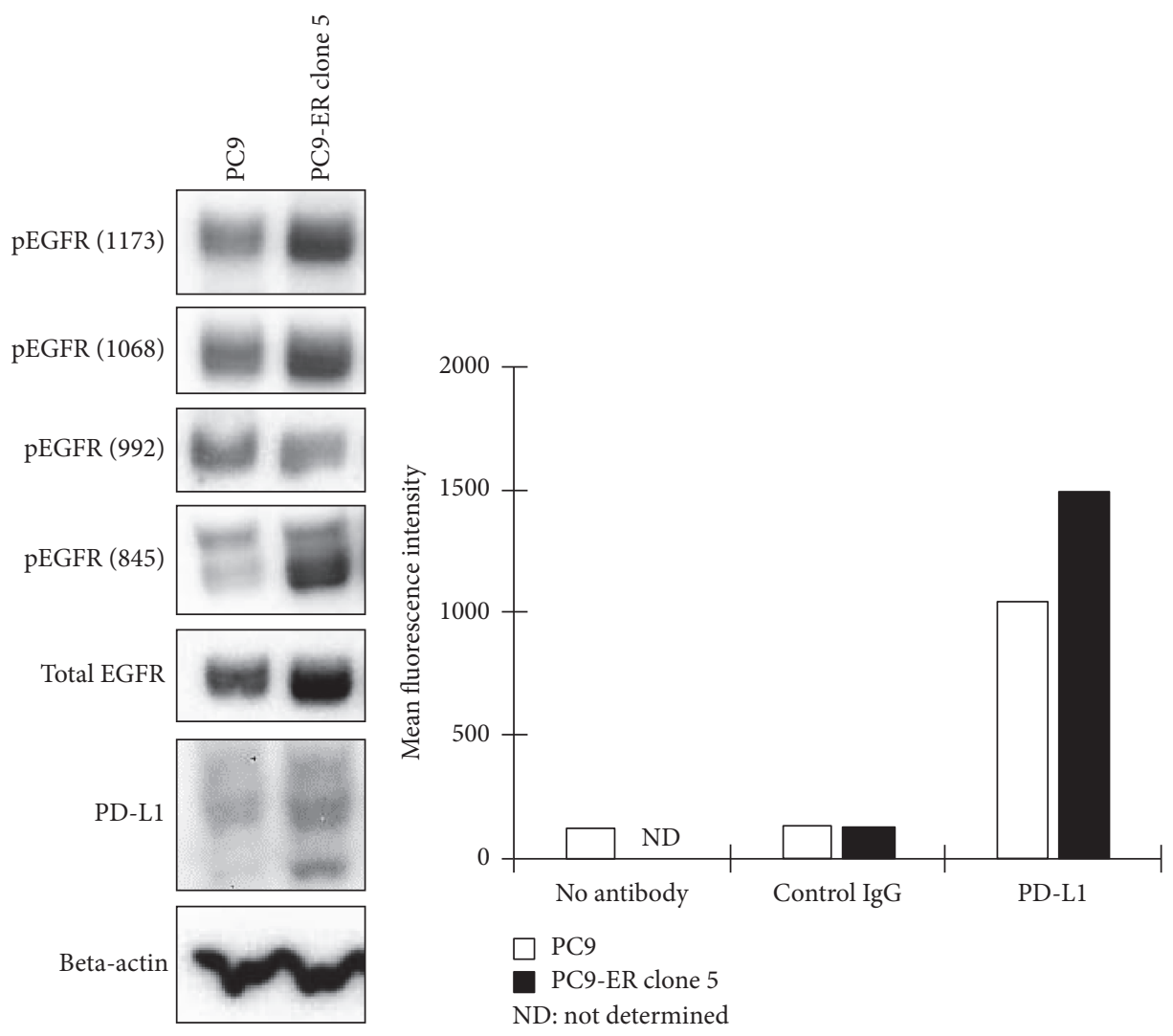

(a)

(b)

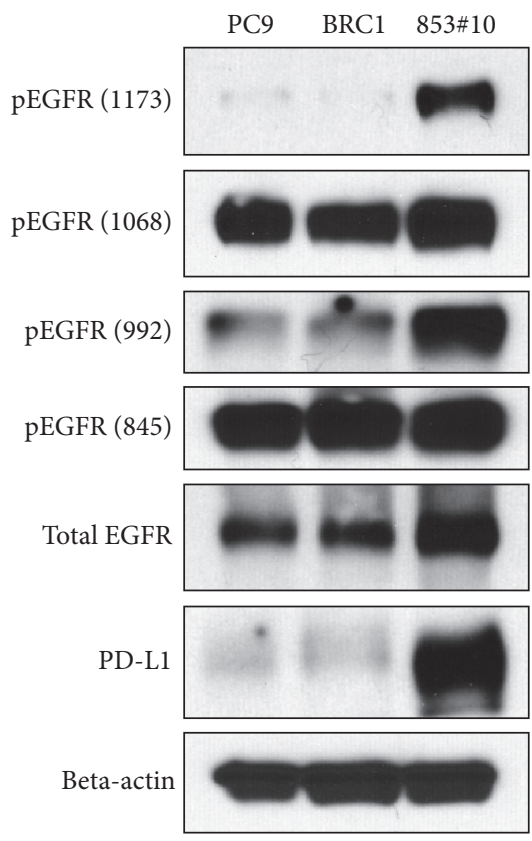

(c)

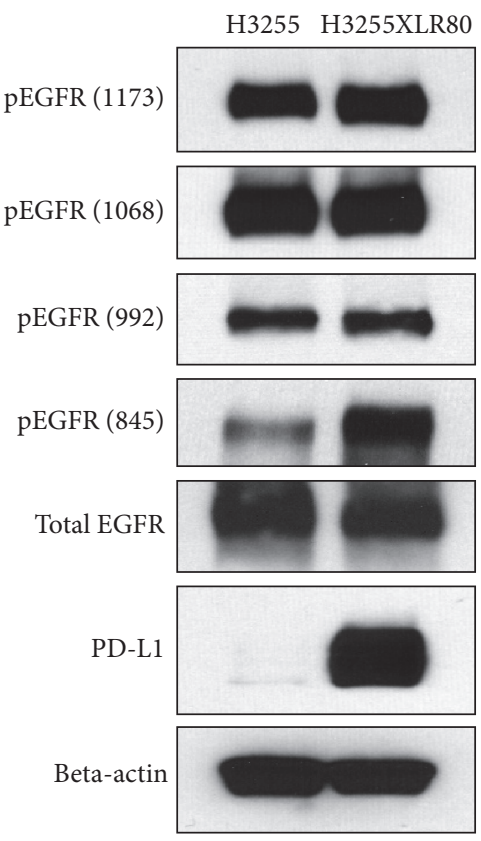

(d)

FIGURE 3: PD-L1 expression and EGFR phosphorylation status in H3255 and PC-9 cells that acquired resistance to EGFR-TKIs. (a) and (b) PC-9 ER clone 5 showed moderate increase of p-EGFRs together with slight increase of PD-L1 expression by western blotting (a) and flowcytometry (b). (c) The other series of PC-9 daughter cell lines resistant to EGFR-TKIs. BRC1 cells showed similar phosphorylation status of EGFR and PD-L1 compared with parental cells, while 853\#10 showed dramatic increase of p-EGFR (Y992 and Y1173) and PD-L1. (d) H3255XLR80 also showed dramatic increase of p-EGFR (Y845) and PD-L1. 
mutation, and the PD-L1 expression decreased when cells developed resistance to EGFR-TKIs by a non-T790M mediated resistance mechanism. In our previous study, we also found that EMT, another non-T790M mediated resistance mechanism to EGFR-TKIs, decreased PD-L1 expression in lung cancer cells with an EGFR mutation [11]. Although acquired resistance cells with increased EGFR phosphorylation (all of them harbored T790M mutation) showed higher PD-L1 expression, osimertinib monotherapy is the current standard treatment for these patients.

Oncogenic signaling within tumor cells and stimuli from the microenvironment both affect PD-L1 expression in tumor cells (constitutive and adaptive PD-L1 expression, resp.). Therefore, our current study looked only at half of the picture (constitutive PD-L1 expression only). However, our results are consistent with clinical findings that showed poorer response rates of $\mathrm{PD}-1 / \mathrm{PD}$-L1 targeting agents in lung cancer patients with EGFR mutations, and may explain at least part of the reasons for the lower efficacy of these agents in these specific patients.

\section{Conclusions}

In summary, we showed that lung cancer cell lines with EGFR mutations do not have high $\mathrm{PD}-\mathrm{L} 1$ protein expression by an FDA approved PD-L1 test. In addition, we demonstrated that PD-L1 expression changes dramatically after acquisition of resistance to EGFR-TKIs, and that was correlated with phosphorylation status of EGFR. Our data implies possible low PD-L1 expression in TKI-refractory lesions without T790M mutation, and that can be one of the molecular mechanisms that attenuates the efficacy of PD-1/PD-L1 targeting agents in lung cancer patients with EGFR mutations.

\section{Conflicts of Interest}

The authors declare that they have no conflicts of interest related to this study.

\section{Acknowledgments}

The study of PC-9 ER clone 5 was funded by Chugai Pharmaceutical Co., Ltd. The other study was supported by an IASLC Young Investigator Award 2015-2017 to K. Suda.

\section{References}

[1] F. R. Hirsch, K. Suda, J. Wiens, and P. A. Bunn, "New and emerging targeted treatments in advanced non-small-cell lung cancer," The Lancet, vol. 388, no. 10048, pp. 1012-1024, 2016.

[2] K. Suda, H. Mizuuchi, Y. Maehara, and T. Mitsudomi, "Acquired resistance mechanisms to tyrosine kinase inhibitors in lung cancer with activating epidermal growth factor receptor mutationdiversity, ductility, and destiny," Cancer and Metastasis Reviews, vol. 31, no. 3-4, pp. 807-814, 2012.

[3] T. S. Mok, Y. L. Wu, M. J. Ahn et al., "Osimertinib or platinum-pemetrexed in EGFR T790M-positive lung cancer," New England Journal of Medicine, vol. 376, no. 7, pp. 629-640, 2017.
[4] K. Suda and T. Mitsudomi, "Role of EGFR mutations in lung cancers: prognosis and tumor chemosensitivity," Archives of Toxicology, vol. 89, no. 8, pp. 1227-1240, 2015.

[5] A. G. Sacher and L. Gandhi, "Biomarkers for the clinical use of PD-1/PD-L1 inhibitors in non-small-cell lung cancer: A review," JAMA Oncology, vol. 2, no. 9, pp. 1217-1222, 2016.

[6] J. F. Gainor, A. T. Shaw, L. V. Sequist et al., "EGFR mutations and ALK rearrangements are associated with low response rates to PD-1 pathway blockade in non-small cell lung cancer: A retrospective analysis," Clinical Cancer Research, vol. 22, no. 18, pp. 4585-4593, 2016.

[7] C. K. Lee, J. Man, S. Lord et al., "Checkpoint inhibitors in metastatic EGFR-mutated non-small cell lung cancer-a metaanalysis," Journal of Thoracic Oncology, vol. 12, pp. 403-407, 2017.

[8] K. Suda, I. Murakami, T. Katayama et al., "Reciprocal and complementary role of MET amplification and EGFR T790M mutation in acquired resistance to kinase inhibitors in lung cancer," Clinical Cancer Research, vol. 16, no. 22, pp. 5489-5498, 2010.

[9] V. Pirazzoli, C. Nebhan, X. Song et al., "Acquired resistance of EGFR-mutant lung adenocarcinomas to afatinib plus cetuximab is associated with activation of mTORC1," Cell Reports, vol. 7, no. 4, pp. 999-1008, 2014.

[10] H. Mizuuchi, K. Suda, I. Murakami et al., "Oncogene swap as a novel mechanism of acquired resistance to epidermal growth factor receptor-tyrosine kinase inhibitor in lung cancer," Cancer Science, vol. 107, no. 4, pp. 461-468, 2016.

[11] K. Suda, L. Rozeboom, C. J. Rivard et al., “Therapy-induced E-cadherin downregulation alters expression of programmed death ligand-1 in lung cancer cells," Lung Cancer, vol. 109, pp. $1-8,2017$.

[12] M. Reck, D. Rodríguez-Abreu, A. G. Robinson et al., "Pembrolizumab versus chemotherapy for PD-L1-positive nonsmall-cell lung cancer," New England Journal of Medicine, vol. 375, no. 19, pp. 1823-1833, 2016.

[13] R. S. Herbst, P. Baas, D.-W. Kim et al., "Pembrolizumab versus docetaxel for previously treated, PD-L1-positive, advanced nonsmall-cell lung cancer (KEYNOTE-010): a randomised controlled trial," The Lancet, vol. 387, no. 10027, pp. 1540-1550, 2016.

[14] F. R. Hirsch, A. McElhinny, D. Stanforth et al., "PD-L1 immunohistochemistry assays for lung cancer: results from phase 1 of the blueprint PD-L1 IHC assay comparison project," Journal of Thoracic Oncology, vol. 12, no. 2, pp. 208-222, 2017.

[15] E. A. Akbay, S. Koyama, J. Carretero et al., "Activation of the PD1 pathway contributes to immune escape in EGFR-driven lung tumors," Cancer Discovery, vol. 3, pp. 1355-1363, 2013.

[16] K. Azuma, K. Ota, A. Kawahara et al., "Association of PD-L1 overexpression with activating EGFR mutations in surgically resected nonsmall-cell lung cancer," Annals of Oncology, vol. 25, no. 10, pp. 1935-1940, 2014.

[17] N. Chen, W. Fang, J. Zhan et al., "Upregulation of PD-L1 by EGFR activation mediates the immune escape in EGFR-driven NSCLC: Implication for optional immune targeted therapy for NSCLC patients with EGFR mutation," Journal of Thoracic Oncology, vol. 10, no. 6, pp. 910-923, 2015.

[18] K. Lin, J. Cheng, T. Yang, Y. Li, and B. Zhu, "EGFR-TKI downregulates PD-L1 in EGFR mutant NSCLC through inhibiting NF- $\kappa \mathrm{B}$," Biochemical and Biophysical Research Communications, vol. 463, no. 1-2, pp. 95-101, 2015.

[19] N. Rodig, T. Ryan, J. A. Allen et al., "Endothelial expression of PD-L1 and PD-L2 down-regulates CD8+ T cell activation and 
cytolysis," European Journal of Immunology, vol. 33, no. 11, pp. 3117-3126, 2003.

[20] M. Rojas, S. Yao, and Y.-Z. Lint, "Controlling epidermal growth factor (EGF)-stimulated Ras activation in intact cells by a cellpermeable peptide mimicking phosphorylated EGF receptor," Journal of Biological Chemistry, vol. 271, no. 44, pp. 2745627461, 1996.

[21] D. R. Emlet, D. K. Moscatello, L. B. Ludlow, and A. J. Wong, "Subsets of epidermal growth factor receptors during activation and endocytosis," Journal of Biological Chemistry, vol. 272, no. 7, pp. 4079-4086, 1997.

[22] K. J. Lastwika, W. Wilson, Q. K. Li et al., "Control of PD-L1 expression by oncogenic activation of the AKT-mTOR pathway in non-small cell lung cancer," Cancer Research, vol. 76, no. 2, pp. 227-238, 2016.

[23] S. Ikeda, T. Okamoto, S. Okano et al., "PD-L1 is upregulated by simultaneous amplification of the PD-L1 and JAK2 genes in non-small cell lung cancer," Journal of Thoracic Oncology, vol. 11, no. 1, pp. 62-71, 2016. 


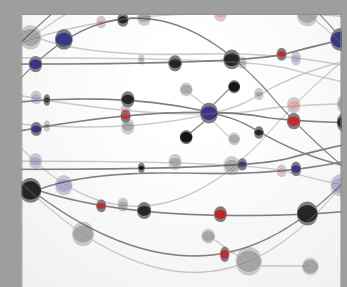

The Scientific World Journal
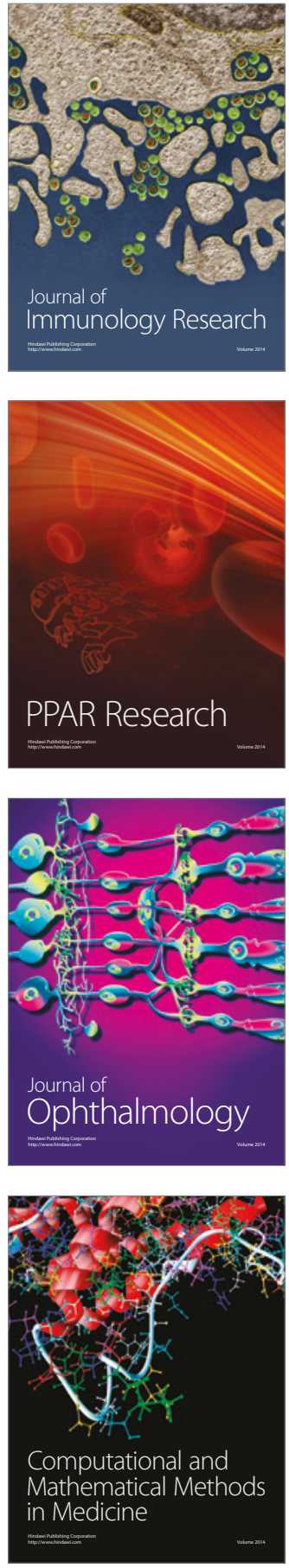

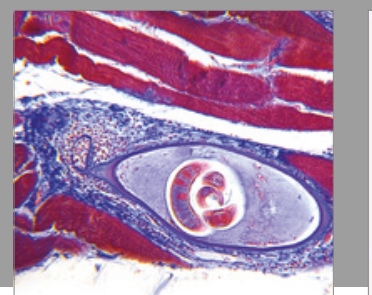

Gastroenterology Research and Practice
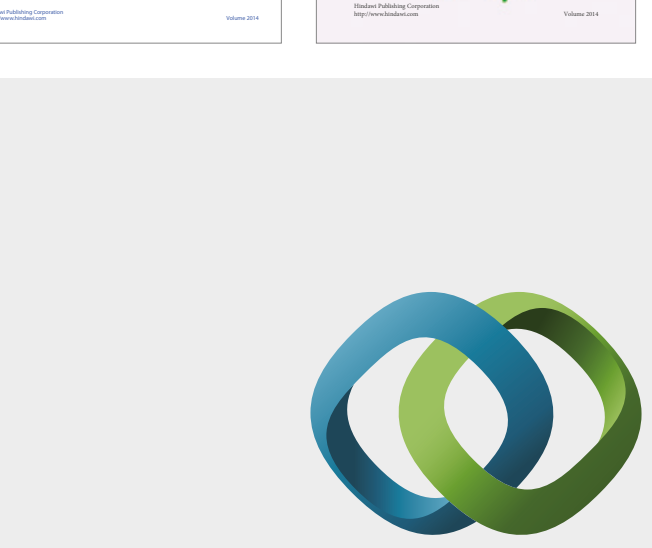

\section{Hindawi}

Submit your manuscripts at

https://www.hindawi.com
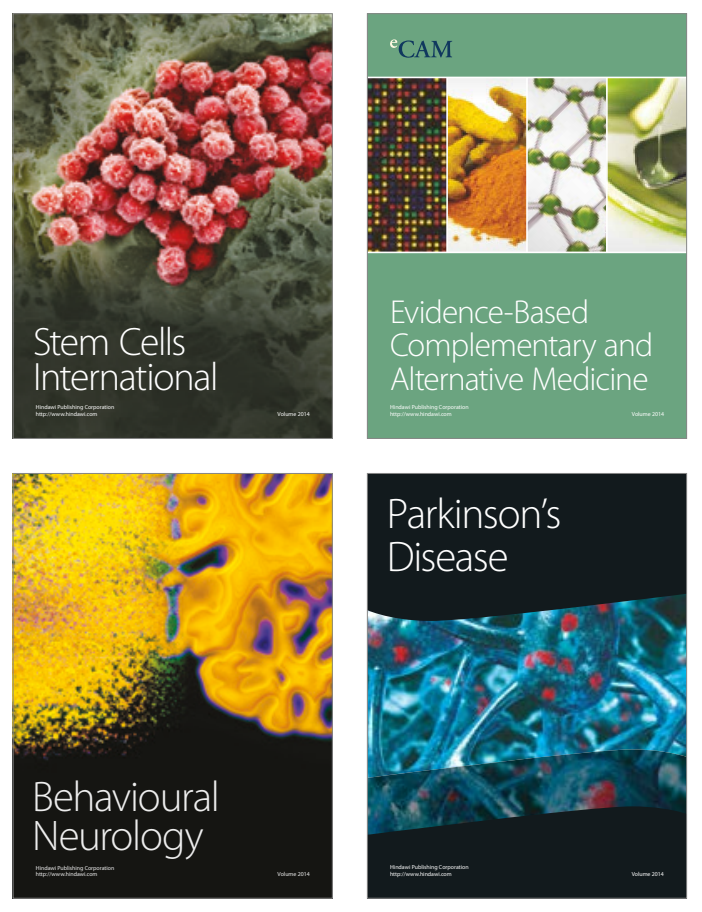
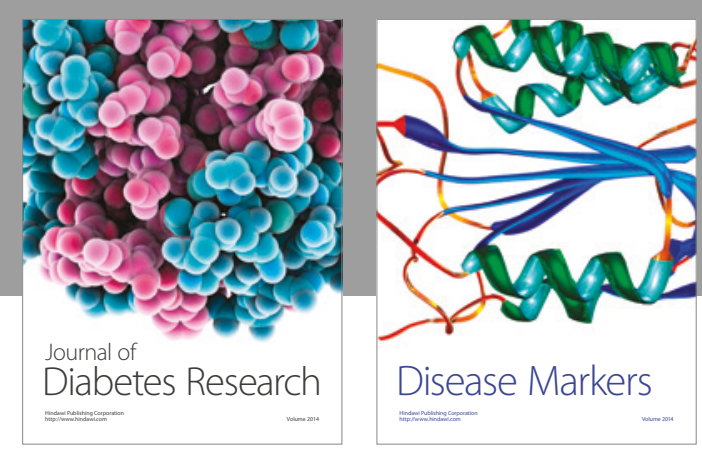

Disease Markers
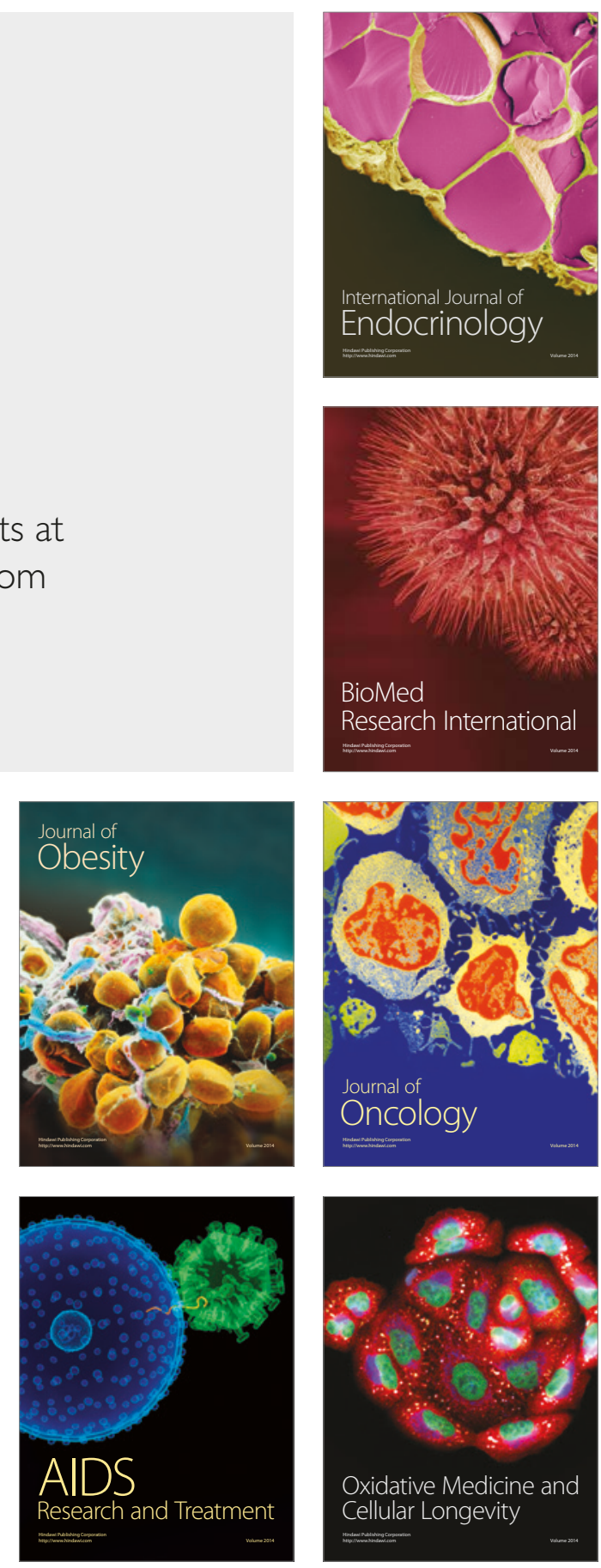